\title{
REPLANTEANDO EL SENTIDO SOCIAL DE LA ESCUELA DESDE LA TRIADA: CUERPO, EDUCACIÓN Y PANDEMIA
}

\section{School and it's Connection with Education, the Body and the Pandemic}

CLAUDIA PONTÓN RAMOS

UNAM (MÉXICO)

PONTON@UNAM.MX

ORCID: 0000-0001-6531-7699

DOI: https://doi.org/10.5565/rev/mitologias.820

vol. 25 | enero 2022 | 38-50

Recibido: 12/09/2021 | Aceptado: 11/01/2022

Resumen:

En este artículo se abordan dos temas centrales, el primero plantea como reto pensar a la escuela y el cuerpo como espacios de aprendizaje; el segundo refiere a pensar en la alteridad del sujeto y su corporeidad como parte de una construcción discursiva y cultural en permanente cambio. La propuesta reflexiva de estos dos aspectos recupera el vínculo entre el cuerpo, la educación y la pandemia como referentes importantes para identificar elementos significativos orientados a resignificar a la escuela como uno de los contextos principales en los procesos de socialización. Con esto, al dimensionar los efectos y consecuencias de la pandemia nos parece necesario proponer cambios relativos a la configuración escolar y su impacto en la construcción de nuevas subjetividades. Este reto reflexivo exige pensar, tanto a la escuela como al ámbito familiar, como escenarios potenciales para reflexionar sobre la noción de sujeto como proyecto, midiendo las consecuencias de un confinamiento forzado, el cual ha provocado una percepción socio-cultural compleja y desbordante. Atendiendo la línea editorial de este dossier, el artículo intenta articular un análisis teórico y cultural de la escuela en tiempo de pandemia. 


\section{Palabras clave:}

Educación, cuerpo, escuela y pandemia

\section{Abstract:}

In this article two central themes are addressed; the first one propounds as a challenge to think of school and the body as learning spaces, while the second one refers to the thought about the otherness of the subject and its corporeality as part of a discursive and cultural construction in permanent change. The reflexive proposal brought up by these two aspects recovers the link between the body, education, and the pandemic as important references to identify significant elements oriented towards re-signifying school as one of the main backgrounds behind socialization processes. Considering the effects and consequences of the pandemic, it seems necessary to propose changes related to the school configuration and its impact on the construction of new subjectivities. This reflexive challenge requires thinking both, the school and the family environment, as potential scenarios to reflect on the notion of the subject as a project, considering the consequences of forced confinement, which has caused a complex and overflowing socio-cultural perception. Following the editorial line of this journal, this article attempts to articulate a theoretical and cultural analysis of the school in times of pandemics.

\section{Keywords:}

Education, Body, School and Pandemic 


\section{Presentación}

Desde el inicio de la pandemia, las dinámicas institucionales relacionadas con las interacciones de la vida escolar fueron drásticamente trastocadas por la necesidad incuestionable de cuidarnos entre todos y todas. Esta disyuntiva modificó de forma significativa no solo lo relativo al vínculo pedagógico, sino también las interacciones entre los actores sociales. El ajuste y acomodo de los vínculos rebasaron a los espacios escolares. La escuela como espacio físico privilegiado para desarrollar el proceso de enseñanza y aprendizaje se desdibujó al transitar hacia el espacio familiar. Este proceso de desplazamiento se dio de manera forzada e inmediata.

Docentes, alumnos/as y los actores del entorno familiar tuvimos que modificar dinámicas y formas de organización tanto del trabajo escolar como de la vida en los hogares. Las condiciones derivadas de la pandemia ocasionaron un trastrocamiento en las dinámicas de organización escolar y familiar. La reconfiguración de los escenarios nos recordó también que la escuela a través de la historia ha representado un dispositivo central para la difusión de los valores socioculturales considerados importantes o hegemónicos. En este marco, el vínculo entre la cultura y la escuela representa un espacio dialéctico y un campo de fuerzas orientado a construir cierto sentido social.

Un aspecto importante, frente a las condiciones de confinamiento ocasionadas por la pandemia, fue replantear la dimensión axiológica de la escuela y priorizar sus funciones éticas y políticas. Lo anterior exige que todos los actores educativos construyan mediaciones analíticas, conceptuales y prácticas, orientadas a configurar a la escuela no solo como parte de una propuesta formativa vinculada con el desarrollo de educación nacional, sino resignificarla también como parte de un proyecto sociocultural que configura procesos identitarios y políticas de reconocimiento, a partir de los cuales es posible reivindicar su función social y su corresponsabilidad ético y política.

Ante estas disyuntivas, la finalidad de este trabajo es reflexionar sobre la función social de la escuela como un espacio potencial para formar subjetividades, a partir de esta triada: cuerpo, educación y pandemia. Consideramos que desde estos vínculos es posible identificar elementos y aspectos analíticos importantes para resignificar a la escuela como un referente central en los procesos de socialización de los sujetos. En este marco de discusión, los planteamientos de autores como Foucault (1984) nos permiten conceptuar a la escuela como un dispositivo de control social al legitimar prácticas de vigilancia y disciplinamiento que contribuyen a la domesticación de los sujetos. Este tipo de argumentos nos exigen analizar de forma autocrítica lo referente a la dinámica regulatoria de las prácticas pedagógicas y dimensionar a la educación como un campo intelectual importante para la construcción sociocultural contemporánea.

En función de este encuadre es fundamental recuperar el debate sobre el sujeto y su potencial formativo, y en paralelo replantear también el papel actual de la escuela y su sentido formativo. La invitación es pensar a la escuela como un espacio de configuración de identidades y subjetividades. Lo anterior nos recuerda que como docentes de cualquier nivel escolar tendríamos que apoyar los procesos de socialización orientados a promover y fortalecer la autonomía del sujeto, así como el desarrollo de una conciencia crítica, histórica y reflexiva, para lo cual es una prioridad compartir como un objetivo común el coadyuvar en la formación de personas autónomas y libres, capaces y dispuestas a defender sus propias ideas.

Dimensionando las consecuencias de la pandemia nos parece necesario proponer cambios relativos a la configuración escolar y su impacto en la construcción de nuevas subjetividades. Recordemos que la escuela forma parte de un entramado social y cultural complejo, el cual da cuenta de una propuesta formativa con impacto social y político, vinculada históricamente a un proyecto más amplio, orientado a contribuir en la formación de una ciudadanía ética y políticamente responsable. Esta constitución de la escuela como espacio público nos obliga a pensar, reflexionar y analizar sobre su relevancia en la configuración de nuevas formas de subjetividad. 
Analizar al espacio escolar no solo como una propuesta formativa relacionada con el desarrollo de la educación nacional, sino también como parte de un proyecto sociocultural orientado a delinear procesos identitarios y políticas de reconocimiento no es solo importante sino necesario, en tanto que replantea ejes articuladores y transversales que posibilitan reivindicar su función social y su corresponsabilidad ética y política. Atendiendo la línea editorial de este dossier, el presente trabajo intenta articular un análisis teórico y cultural de la escuela en tiempos de pandemia.

Es importante identificar que este reto reflexivo nos exige incorporar una lectura transdisciplinaria, a partir de la cual sea posible interpelar tópicos poco abordados en nuestro campo conceptual y de intervención pedagógica y educativa. No podemos desconocer la presencia e influencia de enfoques filosóficos, sociológicos, psicológicos e históricos que retoman el tema de la educación (griega, renacentista, moderna, etc.), y que derivan en análisis y debates concretos sobre sus procesos de institucionalización y conceptuación.

En el marco de este trabajo se abordarán dos ejes de interpelación: el primero plantea como reto el pensar a la escuela y el cuerpo como un espacio de aprendizaje; el segundo refiere a pensar en la alteridad del sujeto y su corporeidad como parte de una construcción discursiva y cultural en permanente cambio.

\section{Pensar a la escuela y al cuerpo como un espacio de aprendizaje}

A través de la historia se ha considerado que uno de los objetivos de la educación en general es enseñar y trasmitir valores, hábitos, costumbres, comportamientos y pautas socioculturales que faciliten el proceso de adaptación de las nuevas generaciones. En conjunto, la familia, las escuelas y los medios de comunicación adquieren un papel central para lograrlo.

Desde el siglo XVIII la educación escolar recibe un lugar central; primero, como un referente de libertad y autonomía, que define el inicio del pensamiento pedagógico moderno, el cual se fortalece con la creación de la escuela pública moderna y la introducción del Método en la pedagogía y en la educación, bajo los principios de racionalidad derivados de la obra de Descartes. ${ }^{1}$ Posteriormente, en siglo XIX, se afianza la idea de la escolarización como un eje rector para el desarrollo social y cultural de la humanidad. A partir de este siglo se empieza a consolidar la pedagogía moderna, experimental, instruccional y por objetivos, que se caracteriza por obedecer al reconocimiento de los ideales modernos y el poder de la ciencia sobre el porvenir de las infancias y de las juventudes.

Para algunos especialistas, desde el siglo XVII la enseñanza se constituye sobre la base de un discurso metódico, capaz de articular una forma de ser maestro, unos procedimientos específicos, un lugar delimitado (la escuela), unos contenidos y una caracterización de la forma de aprender (didáctica) (Zuluaga y Echeverri, 2003: 33). Se enfatiza también que desde los griegos la paideia se articula con la educación a partir de tres principios: valor, lucha y virtud. En este sentido, educar implicaba la idea del ser y estar en el mundo (Quinceno, 2011: 31-32). Este planteamiento original de la paideia se sustituye por las nuevas configuraciones que se construyen sobre la escuela, soportadas por las doctrinas morales,

\footnotetext{
${ }^{1}$ Sobre este tema, Humberto Quinceno plantea que Descartes separó el mundo del pensamiento y la realidad, al sostener que el pensamiento es una representación mental de la realidad. En la filosofía cartesiana el sujeto se presenta como una condición absoluta de la existencia de las representaciones sobre el mundo. Para Quinceno, Comenio se apoya en esta idea para construir la didáctica y la escuela (2011: 50-51). En este marco, la educación se configura como una representación mental que está compuesta de instituciones, discursos y sujetos.
} 
derivadas de las comunidades religiosas, el desarrollo de la lógica jurídica y el diseño de las políticas estatales. $^{2}$

En la época medieval, la educación se vincula con la providencia divina y sus principios morales, y con la lógica aristotélica. En cambio, con la influencia de Descartes y el desarrollo del método científico, la educación se relaciona con una identidad racional y se plantea la idea del sujeto educado desde principios objetivos a partir de los cuales se desarrollaron tecnologías, metodologías de investigación y procesos de institucionalización, fortaleciendo el vínculo entre el sujeto educado y la sociedad moderna. En el siglo XVIII, con los planteamientos de Descartes y Rousseau, se vislumbra la categoría de ciencia para el abordaje tanto de la educación como objeto de estudio como de la pedagogía como disciplina. Ya para finales del siglo XIX y principios del XX se establecen instituciones sociales y políticas que exigen una configuración diferente de la educación y se instituye un vínculo directo entre la educación y la formación de la ciudadanía.

A partir de este tipo de prescripciones, la escuela empieza a configurarse como una institución disciplinaria orientada a los controles legales, administrativos y morales, enlazados a un proyecto de Estado y de legislación, soportados por procesos de institucionalización. Estas herencias históricas nos exigen analizar el efecto de sus dispositivos para la configuración de otras subjetividades.

Los efectos y consecuencias de la pandemia provocada por la COVID-19 nos han dejado aprendizajes colectivos sobre la fragilidad de la vida humana en general. Esta condición se convierte también en un detonante para replantearse muchos ámbitos ligados con nuestra vivencia cotidiana. Uno de ellos son los espacios escolares y familiares como referentes importantes de socialización y configuración de nuevas identidades y procesos de subjetivación.

En la literatura especializada sobre el tema, la subjetividad refiere a un campo problemático en donde confluyen imaginarios colectivos, representaciones sociales, memorias, creencias, ideologías, saberes, sentimientos, voluntades y visiones de futuro; todos estos aspectos permiten configurar un sentido de pertenencia a un nosotros y al conjunto social. En este marco se enfatiza que la subjetividad, como producción simbólica y situada históricamente, es siempre alteridad indeterminada y transversal, la cual se cristaliza en instituciones, normas, costumbres, rituales y modos de hacer (Torres, 2006: 91-92). Se enfatiza, también, que los individuos son siempre sujetos situados en relaciones múltiples y heterogéneas, condición que nos exige abordar a la subjetividad como una dinámica constituyente, la cual oscila de forma permanente entre las dimensiones micro y macrosociales (Zemelman, 2010).

Sobre este punto es importante recordar que dentro de la historia de nuestro ámbito de estudio la infancia se institucionaliza con el apoyo de la psiquiatría, la medicina y el derecho. Desde estas lógicas, las escuelas, las instituciones médicas y las instancias jurídicas desarrollan programas y acciones encaminadas a la regulación de las prácticas y los cuerpos de los sujetos. Un tema de debate permanente que da cuenta de este tipo de influencias es el ejercicio de la sexualidad; tópico en el que desde sus inicios, tanto los dispositivos pedagógicos, médicos y jurídicos, de forma articulada, han introducido ciertos procesos de subjetivación en torno al deber ser de la sexualidad, definiendo a través de la historia procesos de regulación del pensar y practicarla.

\footnotetext{
2 En 1823, en México se delinea el primer proyecto de Reglamento General de Instrucción Pública, en donde se explícita que la instrucción debe ser pública, gratuita y uniforme; sin embargo, es hasta 1867, en la llamada República Restaurada, cuando se sientan las bases del sistema educativo. Un sistema que utiliza al proceso de Enseñanza y Aprendizaje como un referente central, orientado a transformar los patrones de comportamiento y agilizar una modernización económica y política. Sobre estos principios, se dicta la Ley Orgánica de Instrucción, que garantizaba una enseñanza gratuita y obligatoria de educación elemental (Galván Lafarga, 2000).
} 
En los diferentes procesos de socialización podemos identificar cómo la actividad del cuerpo se encuentra sometida a distintos regímenes institucionales, los cuales establecen los códigos de acción tanto en lo laboral, religioso, familiar etc. Referentes del análisis de lo corporal como un dispositivo de poder frente a las ideas normativas del castigo, o el desarrollo de prototipos femeninos considerados perfectos, debido al impulso de nuevas tecnologías orientadas a la trasformación de la fisonomía femenina, mediatizada por el impacto de estándares soportados por la llamada "belleza artificial"; el lenguaje del cuerpo y sus símbolos, frente a los consumos culturales que reafirman la lógica patriarcal binaria; la existencia, por ejemplo, de una negación de las corporalidades lésbicas o de hombres gay o transexuales frente al esquema de la ciudad heterosexual. Todos estos aspectos reproducen actitudes sexistas y homofóbicas de discriminación y exclusión, que se repiten e incluso se radicalizan tanto en los escenarios familiares como escolares.

Si realmente trabajamos para formar ciudadanos éticamente responsables, es central reflexionar como docentes sobre las iniciativas que estamos desarrollando para combatir estos escenarios. Con independencia de las particularidades de los ámbitos disciplinarios es fundamental establecer una mediación analítica entre los contenidos de nuestros programas escolares y nuestros entornos sociopolíticos, culturales y geográficos. Los docentes, como actores centrales del vínculo pedagógico, no podemos quedarnos al margen de problemáticas compartidas y emergentes por ser atendidas, como la exclusión y la equidad en la educación. Ambos son aspectos que preocupan a todos los sistemas educativos en el mundo. No podemos olvidar, por otra parte, que el problema de la equidad se vincula de forma directa con el de la igualdad de oportunidades para todos y todas.

En este marco, la perspectiva de género dentro del ámbito educativo adquiere un lugar importante, en tanto que permite configurar una analítica centrada en la igualdad, la libertad y el equilibrio (cultural, social, político, económico, etc.) de todas y todos los actores educativos, estableciendo ciertos mecanismos de regulación que minimicen las desigualdades construidas tradicionalmente en las estructuras sociales.

Sobre este tipo de argumentos, resulta central resaltar la relación dialéctica entre sexualidad, erotismo y cuidado del cuerpo, vistos como recursos para analizar la construcción de subjetividades. Turner (1989) plantea que existe un nexo entre el régimen de una sociedad y las regulaciones que obran sobre lo corporal, estableciendo una autorregulación social del cuerpo mediante diferentes representaciones sociales.

La historia nos aporta elementos interesantes sobre el tema, por ejemplo, nos ayuda a entender que las percepciones sobre lo corporal son producto de un entorno sociocultural y de momentos históricos determinados. Para algunos autores, el cuerpo femenino en la segunda mitad del siglo XIX estuvo marcado por su capacidad reproductiva, pues la maternidad se convirtió en el eje que definió las expectativas sociales que se tenía de las mujeres y las políticas de Estado. En la literatura sobre esta temática se reconoce que este modelo de maternidad tradicional se ha conceptualizado desde los estudios feministas como parte de una construcción hegemónica que ha prevalecido por más de dos siglos (Méndez, 2015).

Foucault, con apoyo de su método arqueológico, devela cómo a partir de las formas jurídicas internas, junto con la biología, la medicina y la psiquiatría, se desarrolla una racionalidad discursiva que se establece en las instituciones escolares para instalar un dispositivo de la sexualidad orientado a regular y disciplinar los cuerpos. Este autor nos proporciona referentes para identificar cómo históricamente las instituciones escolares se configuran como espacios de institucionalización y normalización de las prácticas destinadas a establecer una forma de vivir la sexualidad mediante su educación, priorizando discursos moralmente aceptables. Sobre esta lógica, la sexualidad transita de ser considerada como un 
potencial erótico a un saber científico, convirtiéndose, así, en un objeto de vigilancia, administración, control y regulación.

En Vigilary Castigar (1984) Foucault da cuenta de cómo la disciplina se perfiló como una estrategia orientada a consolidar una individualidad dócil, autómata y subordinada, adquiriendo un poder muy sutil pero efectivo en los procesos de constitución subjetiva. En este sentido, desde los planteamientos críticos de este autor, el sujeto se piensa y se vive como efecto de la historia y del poder. Sus aportes y múltiples cuestionamientos evidencian las formas en que el poder actúa a través de las prácticas discursivas en todos los ámbitos de la vida humana.

La configuración del conocimiento como poder y su influencia en los campos discursivos permiten comprender aspectos complejos como la autorregulación de los individuos; la constitución de la subjetividad como un efecto de las relaciones de poder; el vínculo indisoluble entre conocimientos, discursos y verdad, una triada que le permite a Foucault problematizar sobre las diferentes prácticas y técnicas de subjetivación (procesos de gubernamentalidad, biopoder, economía política del cuerpo y tecnologías del yo, entre otras.)

Desde esta lógica, la condición de sujeto responde a una sujeción, a un orden del cual no es posible escapar, así como de un legado histórico que deriva de la cultura y se fortalece por los procesos de socialización. En este marco, la escuela y la educación adquieren un papel muy importante. Desde la perspectiva arqueológica y genealógica de este autor es posible identificar las particularidades de las estructuras culturales mediante las cuales los individuos derivan en sujetos.

Para Foucault (1984), el sujeto responde a pensamientos dogmáticos y conductas establecidas y aceptadas cultural y socialmente. Esta situación reduce su capacidad de pensar por sí mismo, convirtiéndolo en una presa fácil de las lógicas de producción y consumo. ${ }^{3}$ De acuerdo con Antonio Curcu, la educación como espacio posible de constitución de subjetividades no puede descuidar las relaciones estructurales que organizan la producción, distribución y consumo del sentido, por cuanto en el interior de ellas se oculta la lógica reproductora que garantiza la funcionalidad de los modelos de dominación como una racionalidad proveedora de sentidos que determinan un tipo de subjetividad (2008: 205-206).

En este marco de ideas, se plantea que la lucha del sujeto actual implica resistirse a las formas de dominio y sujeción ejercidas desde el poder y también desde los saberes que nos atan a una identidad estigmatizada, destacando que la apuesta ética de Foucault implica que la subjetividad debe proclamarse como derecho a la diferencia, a pesar de todas las limitaciones y restricciones de la realidad (Seoane, 2012: 103). En estos procesos se resalta que la maquinaria escolar cumple con un rol preponderante en la constitución del sujeto moderno y de la modernidad (Quiroga, 2017: 225). En el mismo sentido, se enfatiza que la escuela ha sido pensada como uno de los espacios socializadores por excelencia del ser humano, en donde se educa para la vida a partir de compartir subjetividades determinadas por diferentes experiencias dadas desde lo cotidiano (Rojas y Arboleda, 2014: 132-133).

Como aspecto complementario a este análisis se señala que asumir al sujeto como una construcción inacabada nos lleva a reconocer que dicha categoría adquiere en la literatura contemporánea dos connotaciones: como algo atado, amarrado a las estructuras de significación, de poder y de producción; y como un individuo que al reconocerse sujeto que, reconociéndolos, se desata de dichos condicionamientos y construye nuevas realidades. Hacerse sujeto presupone autorreflexividad, imaginación y capacidad de acción deliberativa. Desde esta perspectiva, abordar lo educativo como un

\footnotetext{
${ }^{3}$ Sobre este tema es recomendable revisar el trabajo de Jorge Zuñiga (2020) en el que se presenta un análisis cuidadoso sobre las implicaciones de autores como Foucault en el marco del desarrollo del pensamiento liberal.
} 
hecho intersubjetivo permite no solo desmarcarse de lo escolar, sino también pensar en la escuela como un escenario de confluencia y de conflicto entre subjetividades (Torres, 2006: 95).

Con respecto al cuerpo podemos identificar varios tópicos que se articulan entre sí. Por una parte, se señala que esta noción en el comienzo del pensamiento y de la historia europea, en la época de la antigüedad griega, no existe. Es a partir de la tradición médico-filosófica que adquiere sentido como un cuerpo individual, unido e integrado (Sampson, 2000: 233). Sobre esta misma línea se resalta que la relación entre el cuerpo y la medicina no es espontánea, sino que se organiza en torno a una serie de prácticas culturales que se naturalizan a lo largo de todo el siglo XIX. Desde este contexto es que la medicina moderna se sostiene sobre una estructura binaria en la cual el cuerpo, la naturaleza y la feminidad se sitúan como objetos de estudio de una mirada racional, masculina y descorporeizada (Del Pozo, 2015: 60-63). Como parte complementaria a este tipo de registros discursivos se pueden identificar también un conjunto de imágenes y representaciones de la mujer, tanto en la literatura como en el ámbito pictórico, que han sido producidas por hombres desde el imaginario de lo masculino; un imaginario que minimiza la acción política y social de las mujeres (Chávez, 1997: 13-23).

A partir de este tipo de herencias culturales y sociales se resalta que tanto los discursos científicos como filosóficos sostienen representaciones culturales sobre lo corporal, que incluso en ciertas épocas justificaban la colonización y esclavitud de los pueblos considerados inferiores y objetos de deseo expansionista europeo. La siguiente cita es muy clara:

La apropiación de los cuerpos de las mujeres negras como fuerza productiva y reproductora por parte del poder colonial y esclavista se apoyó de forma crucial en la cosificación de dichos cuerpos en el plano simbólico, a través de prácticas que construyeron la diferencia cultural y su manifestación más visible, la "raza" como Otra inferior, primitiva y, por lo tanto, susceptible de ser dominada por una civilización eurocristiana superior. (Bringas, 2010: 116)

Recuperando estos argumentos, nos parece central reflexionar sobre el encierro físico y emocional que, por consecuencia de la pandemia, ha obligado a que niños/as, jóvenes y adultos se mantengan en confinamiento. Entendemos que existe una multiplicidad de formas de ser niño/a o joven, así como dinámicas familiares diversas, con lo que es imposible establecer, o al menos delinear, una caracterización de esta problemática. Lo que nos interesa, sin embargo, es pensar tanto a la escuela como al ámbito de interacción familiar a la manera de escenarios potenciales para reflexionar sobre el sujeto como proyecto, dimensionando las consecuencias de un confinamiento forzado, el cual ha producido una asintonía sociocultural compleja y desbordante.

Como profesores, nos enfrentamos a nuevos retos, entre los cuales figuran: el aprendizaje en espacios de confinamiento, la preparación en red, la cultura de Internet, las nuevas brechas digitales, las plataformas de apoyo como sistemas de control delegado, la incertidumbre permanente y los diferentes modos de presencia, la necesidad de construir un sentido de continuidad sin presencialidad, la intención por establecer asimetrías de igualdad ante escenarios de aprendizaje tan desiguales, entre otros más.

Con la pandemia se visibilizaron problemáticas que, si bien ya se habían identificado por la comunidad educativa en general, se enfatizaron, como por ejemplo: el peso de las desigualdades territoriales, geográficas y socio-económicas vinculadas con los avances tecnológicos; las desigualdades de género, develadas en cifras alarmantes sobre violencia sexual y doméstica; la discusión sobre la priorización de los contenidos y el replanteamiento de metodologías orientadas a fomentar la participación de los y las estudiantes y la construcción de nuevos conocimientos y dinámicas de trabajo escolar, frente a las inercias estructurales de las escuelas. En general, la emergencia de las clases virtuales replanteó el problema referente a la capacidad del potencial dialógico de todos los actores educativos. 
De forma paralela, la inclusión y el sentido de corresponsabilidad de los actores educativos alude también a la construcción de una crítica seria y sistemática de perspectivas pedagógicas y didácticas que centralizan su enfoque en criterios prescriptivos, normativos y explicativos sobre el quehacer y actuar educativo, así como a una valoración crítica que permita transitar hacia perspectivas más reflexivas y autogestoras, orientadas a recorrer las fisuras y las fronteras de lo normativo. Nos referimos a posturas que rebasen el carácter general y universalista de lo educativo y le den espacio a otro lugar de enunciación, como lo son el transfeminismo, postfeminismo, las teorías queer, los estudios lésbico-gay, las dinámicas de las familias homoparentales, etc.

La irrupción de los y las docentes en el ámbito familiar de nuestros estudiantes nos exige, también, replantearnos el vínculo sujeto-sujeto. Esta no es solo una cuestión que ha de plantearse bajo la pregunta ¿cómo pensar a la escuela sin la presencialidad?, sino también, ¿cómo evitar volver a lo mismo?

Todo lo anterior generó una multiplicidad de preguntas compartidas tanto por los profesores, como el estudiantado y los integrantes del entorno familiar: ¿cómo provocar y generar una discusión productiva en las llamadas clases virtuales?; ¿cómo promover la interacción?; ¿cómo interpretar el pensamiento y el sentir de los y las estudiantes frente a la diversidad de realidades y necesidades?; ¿qué significa ver las cámaras apagadas?; ¿cómo pensar el proceso de adaptabilidad de los y las profesores y alumnos/as frente a la emergencia de lo virtual?; ¿qué currículum necesitamos y cómo lo humanizamos?; ¿qué significa aprender y enseñar en el contexto digital, aún cuando no se considera como un escenario real y confiable? entre otras muchas interrogantes más.

Lo anterior permite identificar que desde la triada cuerpo, educación y pandemia es posible reconocer que, para compartir y generar condiciones mínimas de igualdad, reciprocidad y respeto como especie humana, es fundamental no olvidar que nuestra naturaleza es múltiple, diversa, contingente y variable. Esta situación nos plantea identificar tanto desafíos como prioridades. Ambos aspectos nos obligan también a pensar en retos, provocaciones, combates, luchas, contiendas e incluso duelos, para los cuales no siempre estamos preparados. Esta pandemia nos coloca en el lugar de las respuestas, de las iniciativas y estrategias a considerar en función de nuestros contextos socioculturales o geopolíticos.

El análisis de nuestras experiencias docentes a partir del confinamiento tendría que funcionar como una herramienta conceptual dirigida a desmontar la idea de una cultura fragmentada, utilitaria y consumista que tiende a objetivar y cosificar al cuerpo como un instrumento al servicio de la lógica del mercado y las dinámicas publicitarias. La incertidumbre permanente derivada de esta condición de confinamiento nos obliga a configurar rutas alternas para fortalecer una educación centrada en la conformación de nuevas subjetividades a partir del principio de alteridad, la búsqueda del entendimiento y el sentido de lo colectivo.

\section{Pensar en la alteridad del sujeto y su corporalidad como parte de una construcción discursiva y cultural}

La alteridad del sujeto y su corporalidad la podemos pensar como un proceso de metamorfosis permanente y constante. Esta idea nos hace recordar que la vida incluye cierta fragilidad. La coyuntura actual no da tregua para negarlo. El escenario existente nos impone como un imperativo la necesidad de replantear los aspectos pedagógicos vinculados con la construcción de un mundo más justo e igualitario, lo cual exige rebasar la demagogia y el academicismo, transitar por las fisuras y las fronteras de lo normativo, e identificar y reconocer las tensiones discursivas y el dislocamiento de los saberes. 
Por otra parte, el cuerpo como una construcción discursiva y cultural se relaciona históricamente con el desarrollo del imaginario colectivo. Un imaginario que reproduce la idea de un cuerpo dócil, maleable, útil y productivo, al servicio de las normas socioculturales y la lógica del mercado. Lo anterior nos recuerda el permanente control del cuerpo femenino y su manipulación simbólica que, desde una lógica patriarcal, reduce a la mujer al papel de madre y cuidadora. Este es un tema que se estudia de forma sistemática desde la década de los setenta, pues ahí el debate sobre el cuerpo femenino y sus diversas representaciones se plantea como un núcleo central de las reflexiones feministas.

Para algunos especialistas, el cuerpo en la historia de Occidente nunca había sido tan manipulado como en los últimos años. En esta línea se resalta que el cuerpo se encuentra sometido a la lógica del mercado; se compra, se vende, se modifica y se ajusta a los fines económicos del sistema productivo capitalista. Este sistema refuerza la idea de un cuerpo dócil, útil y productivo, al servicio de las normas sociales y la lógica del mercado, un cuerpo maleable y sometido a los estándares de belleza que domina en el imaginario colectivo (Urzainki, 2015: 297).

Por otra parte, la lógica binaria acentúa ideas que refuerzan las estructuras patriarcales como, por ejemplo, pensar o creer que los hombres son seres racionales, calculadores o fríos, en contraposición de las mujeres identificadas como emocionales, inseguras e impulsivas. O seguir creyendo que el ámbito privado es propio de las mujeres y el público de los hombres; o pensar que hay carreras masculinas y otras femeninas. Entre los efectos negativos de este tipo de estereotipos y prejuicios resalta la estructura de inequidad naturalizada entre mujeres y hombres. Lo anterior es preocupante, pues como docentes de cualquier nivel escolar tendríamos que apoyar los procesos de socialización orientados a fortalecer la autonomía del sujeto, así como el desarrollo de una conciencia crítica y reflexiva.

No podemos desconocer, por ejemplo, que el debate acerca de la educación sexual en las escuelas lleva décadas y aún sigue generando conflictos y grandes controversias derivadas de nociones de sexualidad, cuerpos, familia, normas sociales y culturales, etc. Aspectos que históricamente han ocasionado conflicto por el creciente y permanente escrutinio y control soportado por criterios y "principios" morales y religiosos. Temas que, por otra parte, por lo general no son prioritarios para el diseño de los diferentes proyectos curriculares de las instituciones escolares. Es común que los contenidos sobre educación sexual en las escuelas soporten aspectos vinculados con la clasificación social y el control de los pensamientos, deseos y comportamiento de los sujetos (niños, adolescentes y adultos) en el marco de un contexto de orientación familiar y moral.

Frente a este tipo de condiciones nos podemos imaginar, por ejemplo, los grandes desafíos que enfrentan los profesores de todos los niveles educativos, cuestiones que se agravan y visibilizan por las condiciones de confinamiento ocasionadas por la pandemia. Solo recordemos la importancia que ha adquirido el tema de la salud mental, emocional y física en la actualidad.

Como comunidad docente tendríamos que replantearnos el tema de las desigualdades educativas como un aspecto complejo que da cuenta de una multiplicidad de problemas, como la inequidad social, la pertenencia cultural, el género, la posición económica, los roles sociales y familiares; todos estos aspectos que influyen de forma significativa en las ventajas y desventajas para el desenvolvimiento de los sujetos en educación.

Un gran desafío en este plano argumentativo es estar dispuestos a rebasar el nivel procedimental, prescriptivo y normativo que prevalece en las dinámicas y estructuras organizativas institucionales y escolares; además de resignificar tanto los saberes pedagógicos y sus diferentes dimensiones didácticas, así como las prácticas y el quehacer de los y las docentes. Tanto profesores, autoridades y familias tenemos que ser conscientes y estar dispuestos para pensar el escenario de incertidumbre como un colectivo. 
En estos momentos es fundamental recordar la tesis referente a la naturaleza dialéctica de la escuela como institución social y remarcar su potencial para introducir cambios importantes y significativos en los procesos de socialización de las y los estudiantes. Ante las exigencias de la estructura escolar, la mayoría de los y las profesoras han centrado su responsabilidad profesional en darle continuidad a la transmisión y evaluación de los contenidos y no en las finalidades y consecuencias de lo que se enseña. La manera en cómo desarrollamos nuestra práctica docente, ante cualquier tipo de coyuntura, refleja nuestro posicionamiento sobre el conocimiento y nuestro estar en el mundo.

Es central, por ejemplo, identificar la incertidumbre que genera para las infancias y juventudes el estar separados de sus iguales, atender la exigencia de continuidad de las actividades escolares de manera inmediata, lidiar con los distractores propios de los entornos familiares, con las fallas técnicas y de conectividad, alterar el ritmo y la interacción de los aprendizajes, atender la sobresaturación de las tareas y actividades, así como lidiar con un desgaste físico, mental y emocional permanente.

Aún en los escenarios virtuales prevalecen las violencias simbólicas, emocionales y gestuales, aún con las limitaciones de la pantalla se escapan diferentes formas de manifestaciones de la violencia. Recordemos que la violencia en las escuelas ha sido un tema importante en las últimas décadas. Actitudes de acoso (ahora llamado ciberacoso), peleas, juegos bruscos, burlas, mentiras difundidas en redes sociales, amenazas, conductas impulsivas, comportamientos agresivos, falta de autocontrol, emociones negativas e incluso la violencia auto infligida, forman parte de las dinámicas cotidianas escolares y familiares.

Es una ingenuidad pensar que con la falta de convivencia escolar desaparecen o se mitigan las envidias, competencias, el sentido de popularidad, la discriminación, las conductas intimidantes, de agresión u hostigamiento que se dan entre los actores educativos; ante este tipo de aspectos, pensar a la escuela como un escenario de conflictividad representa otro gran desafío. Un nuevo desafío que nos exige pensar, por ejemplo, en las violencias sexuales o simbólicas de los cuerpos confinados, en la adaptación de las viviendas y espacios familiares en el marco del confinamiento, en el uso del tiempo y el espacio, las modificaciones en las interacciones familiares, los roles de género, las estrategias de subsistencia individual y familiar, entre otros aspectos más.

Por otra parte, pensar el cuerpo desde la alteridad implica observar, identificar y conocer las diferentes formas en que los seres humanos hacemos uso de nuestros cuerpos, y preguntarnos: ¿por qué los marcamos, los modelamos, lo modificamos, lo estilizamos o incluso, lo mutilamos o tratamos con gran indiferencia? Para algunos expertos, la corporalidad y el juego simbólico de la belleza adquieren particularidades y características en cada época cultural, lugar o comunidad (Aviña, 2015: 237). Enfatizan que, en el marco más complejo de la socialización, la actividad del cuerpo se encuentra sometida a distintos regímenes institucionales, los cuales abarcan el ambiente laboral, familiar, religioso, intelectual, entre otros, resaltando cómo una importante vertiente de la representación tecnológica del cuerpo se deriva por bajo el fenómeno de la virtualización (Oliva, 2015: 273).

Este tipo de discusiones o disertaciones conceptuales nos dan herramientas importantes para nuestro accionar como docentes; nos permiten, por ejemplo, minimizar o erradicar la reproducción de conductas homofóbicas, bloquear la hegemonía masculina y patriarcal, identificar los peligros de los escenarios digitales y las redes socio-digitales, establecer un vínculo entre los espacios y las relaciones sociales, cuestionar las jerarquías establecidas por una lógica binaria desigual para hombres y mujeres, recuperar el miedo y la soledad como una preocupación compartida, pensar la idea del cuerpo y la corporalidad como materia y forma de modelar subjetividades.

De acuerdo con Alfredo Marcos (2018), por lo regular tenemos la sensación de que la tecnología nos resulta tan útil como peligrosa y que esta doble condición produce inquietud y desazón. Frente a esto, considera que la noción de "silencio tecnológico" puede funcionar como un puente entre la especulación filosófica y la praxis efectiva. Para este autor, desconectarse de forma voluntaria de las redes 
sociales y de los instrumentos digitales puede fortalecer el desarrollo de virtudes, como la fortaleza, el coraje, la generosidad, la austeridad o la templanza; todos, aspectos fundamentales para bloquear o minimizar de cierta forma el impacto de la tecnología en la modificación de las relaciones espaciotemporales, en nuestros gustos, sueños, experiencias sensoriales, sociales, afectivas y en todos nuestros ámbitos tanto privados como públicos. Lo anterior es importante si recordamos que las tendencias de desarrollo humano en las últimas décadas se han vinculado con la lógica computacional y la credibilidad de los algoritmos. Quizás la propuesta de hacer efectivo el llamado "silencio tecnológico" nos permitirá analizar el impacto y valor epistémico de la llamada cultura tecnológica y sus efectos de corto, mediano y largo plazo.

\section{Bibliografía}

AviÑa CERECER, Gustavo (2015), “Lo que Dios no ha hecho: cuerpo y belleza transexual”, en Rodríguez Cabrera, Verónica; Muñiz, Elsa y Mauricio List (coords.), Prácticas corporales en la búsqueda de la belleza. Ciudad de México, Universidad Autónoma Metropolitana, La Cifra editorial, pp. 235-270.

BRINGAS LÓPEZ, Ana (2010), “Obscuros objetos de deseo: construcciones culturales del cuerpo femenino negro en el discurso publicitario”, en Martín Lucas, Belén (ed.), Violencias (In) visibles. Intermediaciones feministas frente a la violencia patriarcal. Barcelona, Programa Universitario de género. Icaria mujeres y culturas, pp. 115-138.

Chaves José, Ricardo (1997), Los bijos de Cibeles. Cultura y sexualidad en la bistoria de fin del siglo XIX. Ciudad de México, UNAM.

CURCU, Antonio (2008), “Sujeto, subjetividad y formación en educación para pensar en otra visión pedagógica de la evaluación”, Revista de Teoría y Didáctica de las Ciencias Sociales, n. ${ }^{\circ}$ 13, pp. 195-216.

FOUCAult, Michel (1984), Vigilary Castigar. Garzón del Camino, Aurelio (trad.). Ciudad de México, Siglo XXI.

Galván, Luz Elena (2000), "Breve historia de la educación en México", en Sergio Cházaro (coord.), La educación en México. Historia, realidady desafíos. Ciudad de México, Editorial México Desconocido, pp. 27-55.

Quinceno Castrillón, Humberto (2011), Epistemología de la Pedagogía. Ediciones Pedagogía y Educación. Santiago de Cali, Colombia.

QuirogA, Alberto Ramiro (2017), "Escuela y producción de subjetividades. El papel de la educación en las sociedades del gerenciamiento y el paradigma de la gestión escolar", Revista latinoamericana de Filosofía de la educación, vol. 4, n. ${ }^{\circ} 8$, pp. 221-235.

MARCOS, Alfredo (2018), “Silencio tecnológico”, SCIO. Revista filosófica, n. ' 15, pp. 157-176.

MARTÍN LUCAS, Belén (2010), "La madre Patria: de las metáforas nacionalistas a la violación como crimen de guerra”, en Martín Lucas, Belén (ed.), Violencias (In) visibles. Intermediaciones feministas frente a la violencia patriarcal. Barcelona, Programa Universitario de género, Icaria mujeres y culturas, pp. 47-67.

MÉNDEZ MuÑoZ, Martha (2015), "Secretos de tocador: el cuidado del cuerpo femenino en la sociedad decimonónica”, en Rodríguez Cabrera, Verónica; Muñiz, Elsa y Mauricio List (coords.), Prácticas corporales en la búsqueda de la bellera. Ciudad de México, Universidad Autónoma Metropolitana, La Cifra editorial, pp. 91-113.

Oliva ABARCA, Jesús Eduardo (2015), "Frente al (ciber) espejo: los enmascaramientos virtuales del cuerpo", en Rodríguez Cabrera, Verónica; Muñiz, Elsa y Mauricio List (coords.), Prácticas corporales en la búsqueda de la belleza. Ciudad de México, Universidad Autónoma Metropolitana, La Cifra editorial, pp. 271-295.

Rojas ARANGO, Bibiana y Rubiela ARBOLEDA GÓMEZ (2014), "La construcción del sujeto político en la escuela",

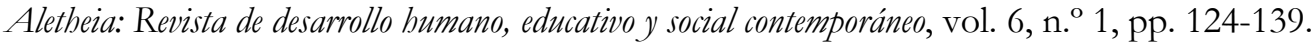


TORRES CARrILlO, Alfonso (2006), "Subjetividad y sujeto: perspectivas para abordar lo social y lo educativo", Revista Colombiana de Educación. Universidad Pedagógica Nacional, n. ${ }^{\circ}$ 50, pp.86-103.

TURNER, Bryan (1989), El cuerpo y la sociedad. Exploraciones en la teoría social. Ciudad de México, Fondo de Cultura Económica.

SAMPSON, Anthony (2000), "Del aula al sujeto: episteme antigua y ciencia moderna en psiquiatría", Revista Colombiana de Psiquiatría, vol. XXIX, n. ${ }^{\circ}$ 3, pp. 231-241.

URZAINKI TOMOS, Maider (2015), "El estadio del espejo: a las órdenes de la imagen estética-ideal del cuerpodelgado (Freud, Lacan, Recalcati)", en Rodríguez Cabrera, Verónica; Muñiz, Elsa y Mauricio List (coords.), Prácticas corporales en la búsqueda de la belleza. Ciudad de México, Universidad Autónoma Metropolitana, La Cifra editorial, pp. 297-326.

ZEMELMAN MERINO, Hugo (2010), "Sujeto y subjetividad: la problemática de las alternativas como construcción posible", Polis, Revista de la Universidad Bolivariana, vol. 9, n. ${ }^{\circ}$ 27, pp. 1-9.

Zuluaga Olga; ECheverRi, Alberto y Humberto Quinceno (2003), Pedagogía y Epistemología. Colección Pedagógica e Histórica. Bogotá, Cooperativa editorial Magisterio.

ZUÑIGA, Jorge (2020), “1978: Foucault y Hinkelammert sobre el neoliberalismo”, Revista Castalia, vol. 34, primer semestre, pp. 109-130. 